Revue internationale P.M.E.

Économie et gestion de la petite et moyenne entreprise

Revue

internationale

PME

\title{
Freins psychologiques et transmission d'entreprise : un cadre d'analyse fondé sur la méthode biographique
}

\section{Philippe Pailot}

Volume 12, numéro 3, 1999

URI : https://id.erudit.org/iderudit/1008657ar

DOI : https://doi.org/10.7202/1008657ar

Aller au sommaire du numéro

Éditeur(s)

Presses de l’Université du Québec

ISSN

0776-5436 (imprimé)

1918-9699 (numérique)

Découvrir la revue

Citer cet article

Pailot, P. (1999). Freins psychologiques et transmission d'entreprise : un cadre d'analyse fondé sur la méthode biographique. Revue internationale P.M.E. 12(3), 9-32. https://doi.org/10.7202/1008657ar
Résumé de l'article

Si le thème de la transmission d'entreprise représente un enjeu majeur pour l'économie française, les freins psychologiques qu'expriment certains dirigeants lors de ce processus de succession managériale n'en restent pas moins mal cernés dans leurs logiques profondes. Sur la base d'une étude de cas d'une TPE familiale, cet article propose un cadre méthodologique (la méthode biographique) et théorique (la psychologie dynamique) susceptible d'éclairer ce phénomène sous un nouvel angle. En nous démarquant du paradigme sociopolitique, nous chercherons à montrer dans quelle mesure l'histoire de vie des dirigeants peut nous renseigner sur les blocages que certains d'entre eux expriment lorsqu'ils doivent quitter leur entreprise. 


\title{
Freins psychologiques et transmission d'entreprise: un cadre d'analyse fondé sur la méthode biographique
}

Philippe PAILOT ${ }^{1}$

Institut d'administration des entreprises de Lille

\section{MOTS CLÉS}

\section{Transmission d'entreprise - Succession managériale Freins psychologiques - Attachement - Méthode biographique}

\begin{abstract}
RÉSUMÉ
Si le thème de la transmission d'entreprise représente un enjeu majeur pour l'économie française, les freins psychologiques qu'expriment certains dirigeants lors de ce processus de succession managériale n'en restent pas moins mal cernés dans leurs logiques profondes. Sur la base d'une étude de cas d'une TPE familiale, cet article propose un cadre méthodologique (la méthode biographique) et théorique (la psychologie dynamique) susceptible d'éclairer ce phénomène sous un nouvel angle. En nous démarquant du paradigme sociopolitique, nous chercherons à montrer dans quelle mesure l'histoire de vie des dirigeants peut nous renseigner sur les blocages que certains d'entre eux expriment lorsqu'ils doivent quitter leur entreprise.
\end{abstract}

\section{L'AUTEUR}

PhILIPPE Pallot est maître de conférences à l'Institut d'administration des entreprises de Lille et chercheur au CLAREE (ESA CNRS 8020 - IFRESI) dans le laboratoire Vie de la Firme. II s'intéresse particulièrement à l'étude des dirigeants de PME, aux relations entre les sciences sociales, l'histoire et les sciences de gestion. Adresse: 104, avenue du Peuple-Belge, 59043, Lille, Cedex, France.

1. L'auteur tient à remercier vivement le quatrième évaluateur pour ses critiques et ses conseils qui lui ont permis d'améliorer la première version de ce texte. 


\begin{abstract}
Even though the transmission of firms is one of the major stakes of the French economy, the psychological drags shown by some company heads during the process of managerial succession are not thoroughly known. Based on the case study of a family Smaller Business Firm (SBF), this article puts forward a methodological frame of study (the biography method) along with a theoretical one (dynamics psychology), which may shed a new light on this phenomenon. On lines other than those of the social and political paradigm, we will try to show to what extent the lives and histories of company heads can inform us about the psychological blocks shown by some of them when they have to leave their business.
\end{abstract}

\title{
RESUMEN
}

El tema de la transmisión de empresa representa una postura mayor para la economía francesa. Sin embargo, los frenos psicolcógicos expresados para algunos dirigentes cuando este proceso de sucesión en gestión de empresa permanecen mal delimitados en sus logicos profundos. Teniendo como base un estudio de caso de una Empresa Muy Pequeña (EMP) familiar, este artículo propone un marco metodologico y teórico capaces de aclarar este fenómeno desde un nuevo punto de vista. Desmarcandonos del paradigma socio-político, procuraremos mostrar cómo la historia de vida de los dirigentes permite comprender los bloqueados de algunos cuando deben separarse de su empresa.

\section{ZUSAMMENFASSUNG}

Auch wenn das Thema der Übertragung einer Unternehmung einen hohen Einsatz der französischen Wirtschaft fordert, wirken die psychologischen Bremsen, welche gewisse Führungskräfte im Rahmen der Unternehmensnachfolge aufweisen, gegen jegliche Vernunft. Auf der Basis einer Fallstudie einer kleinen Familienunternehmung behandelt dieser Artikel einerseits einen methodischen (die biographische Methode) und andererseits einen theoretischen (die dynamische Psychologie) Ansatz, mit dem Ziel, dieses Phänomen von einem anderen Gesichtspunkt aus zu erklären. Vom sozialpolitischen Paradigma distanziert, wird versucht aufzuzeigen, inwiefern die Lebensgeschichte der Führungskräfte Auskunft gibt über deren Blockaden beim Verlassen der Unternehmung.

\section{Introduction}

Dans la littérature sur la transmission d'entreprise, l'analyse des freins psychologiques des dirigeants est certainement l'un des thèmes le plus évoqué, mais le moins étudié dans ses logiques profondes. Trop souvent limités à l'analyse sociopolitique, les modèles explicatifs proposés occultent le plus souvent l'influence de l'histoire de vie des dirigeants qui représente pourtant un facteur structurant central du lien dirigeant-firme auquel toute transmission met un terme définitif. Après avoir rappelé les enjeux de la transmission d'entreprise et souligné les limites du paradigme 
sociopolitique, nous proposerons un cadre d'analyse de ces blocages fondé sur la méthode des récits de vie et s'appuyant sur une analyse du lien dirigeant-firme.

\section{La transmission d'entreprise : enjeux et contexte}

Quand on cherche à établir une hiérarchie des difficultés éprouvées par les PME françaises, l'insuffisance des fonds propres et la transmission sont deux des problèmes les plus souvent évoqués (Hirigoyen, 1987). Si ce second thème n'est pas nouveau (Gélinier et Gaultier, 1974), il n'en reste pas moins d'une actualité aiguë dans les préoccupations des gestionnaires et des gouvernements, et ce, pour au moins deux raisons :

- tout d'abord, dans un horizon de 10 ans, 55000 PME françaises devront remplacer leur dirigeant (Duchénaut, 1995). Or, la majorité de ces dirigeants déclarent ne pas avoir d'héritier pour reprendre leur affaire (Hirigoyen, 1987).

- ensuite, selon une étude du CEPME, plus de $10 \%$ des dépôts de bilan (soit près de 7000 par an en France) seraient la conséquence directe d'une succession effectuée dans de mauvaises conditions (Duchénaut, 1995). Ce constat n'est pas neutre puisque les transmissions ratées seraient à l'origine de la disparition de près de 50000 emplois chaque année.

En première analyse, l'examen du contexte d'une transmission peut être rapproché de plusieurs facteurs explicatifs : l'internalisation du marché des capitaux, qui se conjugue parfois avec celle du marché du travail, contribuant largement à «la mise en place du piège interne » (Vatteville, 1994), l'opacité et la confidentialité du marché de la transmission des PME très éloignées des conditions de la concurrence (Vatteville, 1994), une fiscalité particulièrement pénalisante pour les héritiers repreneurs (Baumert, 1992; Voydeville, 1992), la solvabilité des repreneurs pas toujours détenteurs d'un patrimoine personnel (Amann et Couret, 1992 ; Lehmann, 1993) et l'absence de préparation de la transmission (Hirigoyen, 1987) sont quelques-uns des thèmes les plus souvent abordés pour comprendre le problème de la transmission d'entreprise.

\section{L'approche sociopolitique et ses limites}

À ces éléments juridiques et gestionnaires, il convient d'ajouter le thème classique des freins psychologiques de certains dirigeants qui se manifestent dans le refus de l'inutilité sociale ou de la perte de leur pouvoir. Ce sujet est souvent cité comme critique. Il n'en reste pas moins peu étudié et mal compris dans ses logiques profondes. Trop souvent cantonné dans des explications de type «sociopolitique », il demeure fortement marqué par la sociologie du pouvoir qui permet de relativiser 
le modèle mythique de la transmission spontanée au profit de celui du despote vieillissant qui s'accroche au pouvoir et dirige son entreprise comme si la question du temps et de l'âge ne se posait pas (Bauer, 1993 ; Baumert, 1992 ; Langlois, 1987). Ce positionnement théorique est justifié en soi. En effet, le dirigeantpropriétaire fait rarement la distinction entre pouvoir et propriété (Baumert, 1992). À ce titre, sa rationalité d' "homo politicus » (Bauer, 1993) ne le pousse guère à abandonner son pouvoir, mais plutôt à le conserver et à le consolider en vue de renforcer son monopole dans la production de l'autorité légitime. En s'inscrivant dans une stratégie d'enracinement organisationnel, le dirigeant peut ainsi s'efforcer de rendre l'exercice de son pouvoir personnel inévitable à la survie de celle-ci (Gomez, 1996 ; Paquerot, 1996).

Malgré son intérêt indéniable, le paradigme sociopolitique ne s'inscrit pas moins dans une analyse fonctionnaliste et utilitariste de la « résistance » des dirigeants, en supposant que ceux-ci s'efforcent de «maximiser » leur satisfaction personnelle par une stratégie rationnelle. La conception «néorationaliste» du pouvoir qu'il véhicule conduit à enlever à ce concept tous ses aspects affectifs et dramatiques pour en faire simplement une notion clé autour de laquelle s'élabore l'analyse du phénomène de résistance (Enriquez, 1991). Ce constat n'est pas indépendant des postulats du fonctionnalisme stratégique pour lequel les jeux d'acteurs ne sont pas considérés comme des effets de structure (Crozier et Friedberg, 1977). En adhérant implicitement au postulat méthodologique selon lequel «l'exercice de la fonction détermine le comportement beaucoup plus que les caractéristiques de l'individu » (Bernoux, 1995), cette conception du pouvoir minimise en effet l'influence des déterminations sociales et psychologiques, l'inertie de l'histoire et des mémoires sur les comportements organisationnels. La légitimité de ce réductionnisme méthodologique dans l'analyse organisationnelle ne permet pas d'affirmer pour autant qu'il soit $a$ priori justifié dans l'analyse des processus de transmission où la dimension affective s'énonce pleinement dans les blocages exprimés par certains dirigeants. Cette remarque nous rappelle que les effets d'intelligibilité des concepts n'échappent pas aux limitations de sens prescrits par la théorie. En ce sens, la vigilance épistémologique doit conduire à subordonner leur utilisation à une interrogation sur les conditions et les limites de leur validité.

Sur ce point, les tenants du paradigme sociopolitique ne nous paraissent pas fournir de clarification du rapport conceptuel pressenti entre les notions de freins psychologiques et de pouvoir dans le processus de transmission. Cette difficulté tient certainement en partie à la multiplicité des extensions sémantiques et conceptuelles de la notion de «pouvoir». Ce terme est en effet « une véritable plaque projective qui peut recevoir [...] des significations multiples sinon contradictoires et qui connote des réalités très diverses. [II] permet ainsi une communication fondée sur des malentendus non explorés et qui sont souvent désirés comme tels. C'est en quelque sorte un concept écran qui permet la communication tout en la masquant » 
(Enriquez, 1991). En laissant une place ontologique au pouvoir qui devient une forme ultime d'explication, les modèles sociopolitiques se contentent parfois de nommer le phénomène qu'ils doivent étudier en croyant l'avoir expliqué. Car il faut reconnaître qu'ils ne permettent pas, tout au moins à notre sens, d'expliquer de façon satisfaisante l'un des éléments explicatifs le plus souvent évoqué pour analyser les freins psychologiques des dirigeants lors d'une transmission, à savoir l'attachement viscéral, charnel, quasi possessif qu'éprouvent certains d'entre eux à l'égard de leur entreprise (Baumert, 1992 ; Gélinier et Gaultier, 1974 ; Gaultier, 1980). En effet, leurs ancrages épistémique et théorique les incitent à interpréter la résistance des dirigeants comme une réponse stratégique à la logique d'action du système dans lequel ils sont placés. Ces approches occultent ainsi les éléments de l'histoire de vie des dirigeants qui pourraient pourtant donner une intelligibilité partielle à leurs comportements de défense et jouer un rôle majeur dans la construction du lien d'attachement dirigeant-firme. Ce constat n'est pas indépendant d'une tradition épistémique qui fait l'objet d'un consensus implicite dans les sciences de l'organisation. En délimitant de manière parfois arbitraire la frontière entre l'homme organisationnel et la personne, on oublie peut-être un peu trop souvent que les acteurs n'abandonnent pas leur histoire au seuil de l'entreprise. Ils la portent en eux et elle structure plus ou moins certaines de leurs logiques d'action, et ce, parfois à leur insu.

\section{De l'analyse sociopolitique à l'analyse du lien dirigeant-firme}

Sans en nier l'intérêt heuristique et théorique, notre hypothèse explicative pour comprendre et expliquer les freins psychologiques lors d'une transmission d'entreprise s'inscrit dans une autre orientation théorique et épistémologique que celle proposée par le paradigme sociopolitique. Selon nous, cette résistance tient moins à la volonté des dirigeants de conserver leur pouvoir qu'à un refus conscient, non conscient, voire inconscient, de perdre un objet d'attachement central dans la construction de leur identité personnelle et sociale, voire de leur étayage narcissique, à savoir l'entreprise. En effet, une transmission correspond parfois au dénouement d'une histoire de vie d'un dirigeant, à la perte d'un lien fortement investi et d'un «petit bout de soi» qui s'exprime dans une expérience de crise douloureuse : «Renoncer à ce qui fut une raison de vivre et se pénétrer de la certitude ultime (la mort) est une décision naturellement perçue comme une rupture.» (Vatteville, 1994, p. 19; Kets de Vries, 1988)

Cette orientation nous conduit à avancer l'hypothèse selon laquelle l'analyse de la transmission doit partiellement reposer sur une étude du lien dirigeant-firme dans une approche historique et mémorielle qui reste aujourd'hui largement embryonnaire et implicite. Elle apparaît d'ailleurs comme un axe de recherche particulièrement fécond dans les PME où le chef d'entreprise éprouve un attachement 
profond envers son entreprise (Baumert, 1992 ; Gélinier et Gaultier, 1974), la perçoit comme une véritable prolongation de sa personnalité et s'identifie totalement à elle (Carland et al., 1984 ; Catry et Buff, 1996 ; Duchénaut, 1996 ; Levy, 1988). Or, s'il est souvent évoqué indirectement ou implicitement, cet axe de recherche apparaît paradoxalement un sujet relativement peu étudié en profondeur dans la littérature sur les PME, les théories du cycle de vie de l'entreprise, les théories de la succession managériale ou de la transmission qui n'abordent pas directement ou évacuent la question du lien dirigeant-firme au profit d'autres dimensions. De ce fait, ces différentes théories ne permettent pas de comprendre pourquoi la séparation firme-dirigeant peut être une source de blocages importants. La cause de ce silence théorique repose moins, à notre sens, sur les limites des capacités d'investigation que sur la nécessité de faire appel à des théories peu mobilisées en gestion, mais pourtant susceptibles de nous éclairer sur l'inertie de l'histoire de vie des dirigeants face à l'épreuve de la transmission.

\section{Problématique de recherche et positionnement épistémologique}

Pour éclairer partiellement ces zones d'ombre, nous avons étudié un cas de transmission d'entreprise dans une TPE familiale entre un père (Fernand) et son fils (Philippe) [Pailot, 1995]. La question qui se posait alors à nous était le choix d'un angle d'analyse pertinent pour étudier les freins de Fernand par rapport à l'échéance de la succession managériale. Ce choix nous est apparu évident. Une transmission d'entreprise est, par définition, un acte qui se traduit par la rupture plus ou moins radicale et définitive d'un lien entre un dirigeant et son entreprise, et ce, à des niveaux multiples (financier, matériel, patrimonial, affectif, identitaire, etc.). Dans cette perspective, la forte résistance de certains dirigeants à l'idée de quitter leur entreprise paraît inséparable de la nature du lien qui les relie à leur firme. Sans enjeux identitaires, affectifs et symboliques significatifs associés au processus de transmission, sans l'hypothèse d'un attachement affectif, symbolique et narcissique, voire fantasmatique, profond du dirigeant à son entreprise, on comprendrait mal les raisons profondes de son « refus » de transmission, qu'il soit actif ou passif.

Dans ce cadre, nous avons cherché à montrer que la nature du lien entre Fernand et son entreprise prenait un sens biographiquement construit au regard de son histoire de vie. Cette orientation nous a conduit à mettre au jour certains ancrages psychiques de Fernand, à entendre et à écouter le processus d'intériorisation des événements psychoaffectifs qui s'étaient déroulés dans sa sphère familiale et pouvaient nous aider à comprendre le sens que l'entreprise avait pris à ses yeux. Pour interpréter le matériel biographique, nous nous sommes référé, pour les aspects qui nous intéressent ici, à différents apports de la psychologie dynamique, et notamment aux modèles relationnels de la théorie des relations d'objet. Nous avons également intégré de manière plus marginale un certain nombre d'apports de la psychodynamique du travail (Dejours, 1987, 1993), notamment pour comprendre 
la dialectique paradoxale du plaisir et de la souffrance s'exprimant dans le lien entre le dirigeant et son activité professionnelle. Ce positionnement théorique pourrait heurter le gestionnaire plus familiarisé aux transferts de concepts avec le champ des sciences cognitives, de la sociologie ou de l'économie. Il n'est pourtant pas surprenant en soi. Car l'étude des freins psychologiques dans le cadre d'une approche biographique qui n'intégrerait pas les apports de la psychologie dynamique aurait non seulement quelque chose d'incohérent sur le plan théorique, mais serait aussi, sur le plan épistémologique, injustifiable. Précisons que cette notion recouvre, pour nous, l'ensemble des mécanismes de résistance et de défense qui permettent à un individu de maintenir l'expérience de sa propre unité (cognitive, identitaire, personnologique, narcissique ou autre). Les mécanismes de résistances procèdent de toutes actions ou paroles s'opposant chez un sujet à la prise de conscience de ses logiques d'action profondes. En suivant Widlöcher (1981), ces résistances peuvent être stratégiques (protection des satisfactions infantiles) ou tactiques (fonction défensive du moi). Les mécanismes de défense, quant à eux, « regroupent un ensemble de fonctions du moi destinées à protéger l'individu contre toutes les formes d'agression provenant du psychisme et du monde réel » (Zaleznik, 1994). Pour préciser les rapports entre ces deux notions, disons que les mécanismes de résistance se rapportent au comportement observable, ceux de défense, à l'idée que l'on se fait du processus qui détermine ce comportement. En d'autres termes, les mécanismes de défense sont responsables des résistances. Leur analyse nous conduit cependant à adopter un positionnement épistémologique en rupture partielle avec une certaine orthodoxie gestionnaire.

En effet, en nous attachant à cerner et à interpréter les facteurs d'inertie comportementale dans le matériel biographique de Fernand, nous nous démarquons des conceptions détemporalisées de l'action et de l'intentionnalité consciente, de la théorie rationnelle de l'action ou encore de l'affirmation au droit à l'action libre ou à la conscience claire de celle-ci. À l'inverse d'une vision explicative qui privilégie le synchronique et le situationnel, le questionnement de l'histoire de vie des acteurs organisationnels permet de comprendre dans quelle mesure leurs logiques d'action ne s'expliquent pas exclusivement par des éléments de contexte, mais portent en elles-mêmes les effets du processus historique de socialisation et les conséquences d'un parcours de vie. Entendons-nous bien : il ne s'agit pas pour nous de prôner une vision mécanique de l'homme mû par des forces inconscientes sur lesquelles il n'a pas de prise, ni de promouvoir les vertus explicatives d'un structuralisme étroit. Mais il convient de reconnaître simplement l'influence des dispositions psychologiquement et socialement constituées, l'inertie de certains facteurs psychiques qui rendent illusoire le recours hégémonique à une forme d'intentionnalité consciente détemporalisée.

Sans nier les vertus «d'une réflexivité pragmatique» (Corcuff, 1995) dans la théorie de l'action, on peut penser, comme nous l'affirme la psychologie 
dynamique et cognitive dans des registres différents ${ }^{2}$, que chacun porte en lui son passé duquel il est plus ou moins dégagé et qu'il tend à actualiser dans ses processus perceptifs et dans sa relation aux autres et au monde. En ce sens, la séparation de la personne et de l'homme social peut se révéler être un postulat méthodologique qui, s'il permet légitimement de délimiter les champs scientifiques, n'en risque pas moins de dénaturer la lecture du réel au profit d'une parcellisation dont il convient toujours de remettre en question les fondements et l'intérêt selon les problématiques de recherche. En d'autres termes, il est clair que l'élargissement épistémologique indispensable à toute analyse biographique n'est pas nécessairement pertinent pour toutes les catégories d'objet scientifique des sciences de gestion. Il doit permettre cependant, lorsque le questionnement scientifique le requiert, de discuter les concepts du point de vue des conditions et des limites de leur validité et d'examiner les conditions épistémologiques de production des connaissances scientifiques pour permettre au chercheur d'entrer dans les arcanes de l'invention. Cette dernière donne tout son sens à la recherche scientifique et permet de dépasser le ritualisme des procédures, la soumission à l'instrument et aux modes de pensée dominants, pour reprendre les termes de Bourdieu (1986), qui peuvent paralyser la recherche: "L'intention de donner au chercheur les moyens d'assumer lui-même la surveillance de son travail scientifique s'oppose aux rappels à l'ordre des censeurs dont le négativisme péremptoire ne peut que susciter la terreur de l'erreur et le recours résigné à une technologie investie de la fonction d'exorcisme.» (Bourdieu et al., 1973)

\section{Stratégie de recherche}

Notre stratégie de recherche se situe au croisement de l'étude de cas (Yin, 1981, 1989) et de la méthode des récits de vie (Clapier-Valladon, 1983 ; Clapier-Valladon et Raybaut, 1993 ; Ferrarotti, 1983 ; De Gaulejac, 1984 ; Legrand, 1993 ; Peneff, 1990 ; Pineau et Le Grand, 1993 ; Smith, 1994 ; Bertaux, 1997). La première de ces stratégies de recherche étant bien connue des gestionnaires, nous ne nous attarderons que sur la seconde. Pour des raisons d'espace, nous ne traiterons pas ici des questions qui entourent la scientificité de cette méthode. Précisons seulement qu'elle est aujourd'hui largement légitimée (mais aussi contestée) au sein des sciences anthroposociales (histoire, ethnographie, sociologie, linguistique, psychologie) et analysée par certains gestionnaires comme une ouverture méthodologique potentiellement féconde dans le champ des sciences de gestion (Wacheux, 1996).

Tout d'abord, qu'est-ce qu' une biographie ? Une biographie peut se définir comme une reconstruction narrative, suscitée par un interviewer, du parcours de vie d'un narrateur qui, comme l'Histoire, s'écrit au présent, c'est-à-dire selon un

2. La psychologie dynamique et cognitive établit d'ailleurs une nette distinction entre les cognitions, les processus et les schémas cognitifs et reconnaît pleinement la pertinence de la notion «d'inconscient cognitif » largement citée dans tous les ouvrages traitant des thérapies cognitives. 
principe interprétatif actuel. Irréductible à l'histoire vécue, le récit de vie s'élabore autour d'une construction à plusieurs étages inséparables : 1) d'un regard du présent sur le passé, d'une mémoire qui produit du sens ; 2) d'une conjoncture biographique contextualisée dans une interaction sociale entre un enquêteur et un narrateur; 3) d'un travail de mise en forme du récit de vie qui ne peut occulter les déformations occasionnées par le passage de l'oral à l'écrit (différences de conventions entre le langage écrit et parlé) ou l'irréductibilité entre l'évocation des souvenirs et les souvenirs proprement dits ; 4) d'un questionnement préétabli indissociable d'une problématique théorique de recherche. Par opposition, une biographie n'est ni un accès direct aux conditions objectives de vie d'un narrateur ni une refiguration objective (au sens positiviste ou réaliste du terme) du temps historique vécu par ce dernier : «ce serait pure illusion que de supposer que le récit, la reconstruction ou reconstitution, est simplement le reflet de ce qui s'est passé » (Aron, 1989).

Au-delà des potentialités théoriques et des limites épistémiques de l'approche biographique sur lesquelles nous ne reviendrons pas ici (Pailot, 1996), la méthode des récits de vie constitue, au regard de notre sujet, une stratégie de recherche qui nous a donné des éléments pour cerner la nature du lien d'attachement entre Fernand et son entreprise dans leur développement biographique. Elle nous a donné accès à des niveaux de discours nous permettant d'avancer des explications prenant en considération les «effets de dispositions ${ }^{3}$ » (Boudon, 1986) d'un acteur pour comprendre ses blocages face à la transmission de son entreprise. Cette notion « d'explication dispositionnelle » ne doit pas s'interpréter au sens déterministe du terme. Comme le note Aron (1989), faire référence à des propositions générales relatives à la personnalité d'un sujet n'est pas un moyen d'expliquer pourquoi une action a été posée. Ici, les dispositions doivent plutôt être vues comme un héritage du processus historique de socialisation qui encadre de façon plus ou moins stricte certaines logiques d'action du dirigeant dans l'espace organisationnel.

Quel a été notre dispositif de collecte et d'analyse de matériel recueilli ? En règle générale, le recueil des récits de vie s'appuie sur l'entretien non directif d'enquête (Blanchet, 1983). Pour notre recherche, nous avons conduit personnellement, en garantissant l'anonymat de nos locuteurs, plus de 25 entretiens de trois à quatre heures chacun avec nos interviewés (Fernand, son épouse, son fils et les plus anciens salariés) sur une période s'étalant du mois de juillet 1994 au mois de mars 1995. Ils ont été enregistrés et retranscrits de manière littérale avant de faire l'objet d'une analyse de contenu à la fois qualitative (analyse thématique et structurale, analyse des contenus manifestes et latents, etc.) et quantitative (comptage fréquentiel des unités d'enregistrement, détermination des cooccurrences et de leurs coefficients de corrélation, etc.) qui nous a permis de renforcer la scientificité

3. Les dispositions désignent ici les mémoires rationnelles et affectives qui échappent à l'horizon de notre conscience tout en structurant notre comportement. 
de notre démarche. Pour augmenter la validité des données, nous avons également croisé les discours (examen de la cohérence de chaque témoignage et de la noncontradiction entre les témoignages) en vue de recomposer de façon la plus fidèle possible les éléments factuels et limiter les biais liés à «l'illusion rétrospective» (Bourdieu, 1986). Enfin, sans impliquer Fernand dans le travail d'analyse et d'interprétation de sa vie énoncée, nous lui avons proposé deux bilans de restitution au cours de notre recherche. Cette démarche d'analyse nous a permis de conserver l'objectivité de la méthodologie d'explication du sens (en maintenant une distanciation avec le sujet) tout en autorisant Fernand à participer à l'explicitation du matériel biographique. Ces présentations orales ont permis, par les réactions suscitées, d'exercer une forme de contrôle et d'affinement de nos hypothèses explicatives, d'effectuer une construction ouverte avec le narrateur. Fernand n'a pas contesté ou remis en cause notre interprétation, sauf sur un point mineur.

L'approche biographique n'en reste pas moins une méthode qualitative pour laquelle les phénomènes analysés ne sont pas mesurables (Mucchielli, 1991). À l'instar des sciences historiques (Aron, 1989) et sociales (Passeron, 1991), elle reste inséparable d'une pratique interprétative pour laquelle il n'existe pas a priori de cadre de référence théorique spécifique (Legrand, 1993). Le chercheur doit en délimiter le contenu tant en fonction de la spécificité de sa problématique théorique de recherche que des ressources dont il dispose selon son itinéraire de formation (Legrand, 1993). Cette méthodologie n'en répond pas moins aux exigences des critères de validation scientifique des recherches qualitatives, à savoir l'acceptation interne, la complétude, la saturation, la cohérence interne et la confirmation externe (Mucchielli, 1991), sur lesquels il ne nous est pas possible de nous étendre dans le cadre de cet article (Pailot, 1995). Notons seulement que ces différents critères permettent d'écarter l'arbitraire dans l'interprétation du comportement d'un acteur. Sans eux, on laisserait la porte grande ouverte à l'égocentrisme et au sociocentrisme de l'observateur et tout deviendrait alors affaire de rhétorique.

\section{Pour une analyse sociopsychique du lien dirigeant-firme}

Notre étude de cas est basée sur l'analyse d'une TPE familiale exerçant son métier dans l'imprimerie de labeur ${ }^{4}$ (Pailot, 1995). La société fut créée en 1929 par le père de Fernand, Robert, sous la forme d'une société en nom collectif. Elle exerce alors une activité dans la typographie manuelle sur un marché exclusivement local et avec

4. L'indicateur Imprimerie de labeur correspond au poste $222 \mathrm{C}$ de la nomenclature d'activités française (NAF). Le champ de l'indicateur Imprimerie correspond aux postes $222 \mathrm{C}$ (imprimerie de labeur), $222 \mathrm{E}$ (reliure et finition) et $222 \mathrm{G}$ (composition et photogravure) de la NAF. Les activités de fabrication de la presse quotidienne ne font pas partie du champ de l'indicateur. Sont également exclues les imprimeries intégrées du secteur privé et du secteur public. L'imprimerie de labeur représente près de $82 \%$ de l'activité totale, contre $7,8 \%$ pour la photogravure et $5 \%$ pour la reliure-brochure. 
une clientèle de particuliers. En 1950, Fernand, sur injonction paternelle, entre dans la société comme apprenti typographe minerviste à l'âge de 13 ans et demi. En 1965, Robert décide de construire une librairie-papeterie accolée à l'atelier qui existe toujours aujourd'hui. Le 22 décembre 1971, Robert, âgé alors de 70 ans, décide de transmettre l'entreprise à ses deux fils, Fernand et Hervé, qui répartissent alors le capital de la société à parts égales. La société devient une SARL, mais Robert conservera le poste de gérant jusqu'à sa mort en 1982. À cette date, leur mère deviendra gérante sans assumer aucune responsabilité effective dans l'entreprise. En fait, dès 1972, les deux frères se répartissent les activités de la firme : Fernand prendra la responsabilité du secteur de l'impression, son frère cadet, celui de la librairie. En 1984, le fils de Fernand, Philippe, intègre la société familiale après un engagement volontaire dans la marine de 10 ans. Il propose à son oncle une association dans laquelle il est prêt à investir ses fonds personnels. Face à un silence qu'il interprète comme un refus, il crée le 21 septembre 1984 la société AOD en sous-louant à son père, selon les termes de l'acte exécutoire, « une pièce à usage d'atelier érigée sur le sol de terrain ». Il investit dans du matériel d'impression offset, de photocomposition et de laboratoire. En 1987, après de multiples péripéties, AOD et la branche imprimerie de l'entreprise familiale fusionnent en une nouvelle SARL dans laquelle la mère de Fernand et son frère n'ont plus aucune participation. La société se modernise et se développe. Progressivement, Fernand se sent décalé par rapport à l'évolution de la firme. En accord avec son épouse, il transmettra la société à son fils, en 1993, à l'âge de 57 ans (qui deviendra alors une $\mathrm{SA}$ ) après d'importants problèmes de santé. Il conservera le titre de p.-d.g. jusqu'en 1997.

Concernant le contexte de la transmission, Fernand avait accepté l'idée qu'il passerait un jour ou l'autre le flambeau à son fils, par ailleurs seul successeur possible au sein de la famille, lui-même ayant beaucoup souffert de l'absence de préparation à la transmission qui avait eu lieu entre lui et son père quelque vingt ans plus tôt. Mais celle-ci a été précipitée par deux séries d'événements : 1) des changements technologiques majeurs dans la filière graphique au niveau des procédés d'impression (passage de la typographie à l'offset) et de préimpression (introduction de la PAO) qui rendaient ses compétences obsolètes au sein de l'entreprise, et 2) l'instrumentation du système de gestion de l'entreprise (systèmes de gestion des coûts, informatisation de la gestion, etc.) qui a fait glissé progressivement les compétences gestionnaires du père vers le fils. Ces deux niveaux de changement ont fortement transformé la réalité et le cadre de fonctionnement de l'entreprise qui est devenue en l'espace de 10 ans une entreprise performante ${ }^{5}$ ayant fortement intégré en amont

5. Pour apprécier le niveau de performance de cette entreprise, nous avons procédé à une analyse de sa rentabilité financière, sa rentabilité économique, son taux de marge nette d'exploitation et son taux de marge nette dans une perspective longitudinale sur 13 exercices (analyse des résultats économiques et financiers de l'entreprise de 1982 à 1994) et comparative sur quatre exercices (comparaison des indicateurs de performance retenus par rapport à ceux de ses principaux concurrents sur une période allant de 1990 à 1993). 
la filière graphique avec des équipements technologiques de pointe au niveau du prépresse (PAO, scanneur couleur, flasheuse), alors qu'elle était une imprimerie en perte de vitesse aux technologies obsolètes en 1984. À cela s'ajoutaient les difficultés financières de sa fille qui avait incité Fernand à céder la totalité de ses actions à son fils pour lui venir en aide (selon la logique du pater familias, Bauer, 1993). Le climat relationnel entre le père et le fils dans lequel s'est effectuée la transmission était sans heurts, c'est-à-dire assez éloigné du modèle du « despote vieillissant » ou de celui du « meurtre du père » (Bauer, 1993). Fernand souhaitait un jour ou l'autre passer la main à son fils... mais pas de manière prématurée. Son fils, Philippe, ne souhaitait pas le voir quitter si rapidement la tête de l'entreprise, car, au moment de la succession managériale, il ne s'estimait pas encore prêt à en assumer la direction. Cette bonne entente entre le père et le fils nous a été confirmée à la fois par les deux protagonistes, l'épouse de Fernand et les différents salariés que nous avons interviewés.

Fernand n'en a pas moins mal vécu cette succession managériale. En fait, il a vécu une crise psychique que nous avons analysée avec la théorie du deuil (Pailot, 1995, 1996). Pour cerner les raisons profondes de cette réaction, nous avons cherché à comprendre la nature du lien de sens entre Fernand et son entreprise. L'analyse de contenu de son discours nous a permis de distinguer deux niveaux essentiels intervenant directement dans l'élaboration de ce lien : la nature des relations affectives avec sa famille (notamment sa mère) et son sentiment d'être dépositaire du destin familial. Il est évident que nous ne présenterons, dans la suite de notre exposé, que les conclusions d'une recherche aboutie dont nous ne pouvons rendre compte ici de tous les détails et développements (Pailot, 1995).

\section{La nature des relations affectives avec ses parents}

Durant son enfance, Fernand a vécu dans un contexte familial perturbant caractérisé par une absence de confirmation affective positive et une violence maternelle physique, morale et psychologique omniprésente, violence particulièrement vivace tant que son père était prisonnier de guerre (de 1939 à 1945) alors qu'il vivait, avec sa mère et son jeune frère, dans des conditions matérielles très précaires qui ont conduit au décès de son jeune frère en 1942.

C'était très violent [...] ma mère me pourchassait pour me frapper violemment [...] Ce sont des souvenirs profonds, car à l'époque j'avais même des marques.

Une des conséquences de cette carence affective se traduisait par l'impression, qu'il conserve encore aujourd'hui, de ne pas être aimé et apprécié par les siens, impression qui se conjuguait chez lui avec un sentiment diffus d'infériorité.

Je ne me suis jamais senti aimé [...] tout ce que je faisais était négatif. Je travaillais 80 heures par semaine minimum et j'étais un fainéant [...] C'est encore dur à mon âge de savoir que vous n'êtes rien pour votre mère, cela fait très mal ! 
J'étais un enfant de l'amour, mais pas un enfant désiré [...] Ma mère ne m'a jamais fait voir un geste de tendresse.

Dans cette situation, l'entreprise, dans sa symbolique, avait un poids d'autant plus important aux yeux de Fernand qu'elle lui fournissait un support compensatoire pour « prendre sa revanche », selon ses propres termes, pour prouver sa valeur aux yeux de sa mère et de sa famille, pour mériter (enfin) l'amour et la reconnaissance de cette mère à la fois aimée et haïe.

Au lieu de m'encourager à bien faire les choses, on me faisait comprendre que je n'étais qu'un bon à rien. Et je savais que c'était l'inverse et il fallait que je le prouve. Ce qui fait que j'ai passé toute ma vie à prouver que je n'étais pas un bon à rien [...] Je crois que si je pouvais lui prouver quelque chose, je le ferais encore.

Cette situation compensatoire prenait d'autant plus d'acuité que la résidence de sa mère se situe, encore aujourd'hui, à quelques mètres de l'entreprise. Cette proximité physique permanente ne pouvait que réactualiser les situations inachevées laissées en suspens qui infiltraient sa vie quotidienne et l'entraînaient dans des comportements répétitifs, dans une recherche bloquante et décalée d'un comblement. En ce sens, la volonté de faire ses preuves renvoyait chez Fernand à un mode de défense contre l'angoisse de ne pas exister, à une demande de preuve d'amour toujours déçue et toujours à renouveler. Cette « fixation à la mère frustratrice » était d'autant plus forte que la blessure narcissique qu'il éprouvait (sentiment d'infériorité, etc.) était profonde.

Cette dépendance affective à l'égard d'une image maternelle idéalisée (dans ce qu'elle aurait pu lui apporter) se conjuguait avec une filiation paternelle difficile à assumer (ombre d'un père charismatique pour lequel il avait des sentiments ambivalents) qui renforçait son désir de prouver sa valeur.

L'ombre de mon père a toujours été une entrave [...] On me fait comprendre encore aujourd'hui que mon père faisait mieux que moi [...] J'avais décidé de faire le métier de mon père pour faire voir à ma mère de quoi j'étais capable.

Fernand admet aujourd'hui que ces deux phénomènes ont constitué un principe organisateur fondamental de son existence. Ils expliquent la place centrale, presque exclusive, de «son » entreprise dans sa vie :

Je crois que ça représentait toute ma vie. Il ne se passait pas une nuit sans que je rêvais de mon entreprise. Je la rêvais toujours meilleure. Il n'y a que peu d'années que je ne rêve plus de mon entreprise.

J'ai vécu avec mon entreprise, j'ai vécu pour elle.

Mon entreprise, je crois que c'était ma famille. J'ai vécu pour mon entreprise comme je n'ai pas vécu pour ma famille (le concept de famille s'assimile ici à ses enfants). 
Je me suis fait plus de mauvais sang pour faire tourner mon entreprise que pour l'avenir de mes enfants. J'en suis persuadé.

C'était devenu le seul objectif de ma vie.

En fait, Fernand entretenait une relation passionnelle avec son entreprise et son activité professionnelle.

La passion, c'est de vivre quasiment un amour pour son boulot et son entreprise.

Ce n'est pas un amour charnel, mais ça y ressemble beaucoup.

C'est peut-être plus facile pour un homme de vivre deux amours en même temps, l'amour de sa femme et l'amour du métier et de l'entreprise, c'est bon. Il y a toujours un palliatif pour l'un ou pour l'autre.

Cette captation se retrouvait dans des rêves nocturnes récurrents qui peuvent s'analyser, dans une perspective phénoméno-structurale (Mucchielli, 1993), comme le prolongement de problématiques existentielles et présentes du rêveur.

Je rêvais de mon entreprise presque toutes les nuits. J'avais deux rêves possibles : mon service militaire en Algérie et l'imprimerie. C'était sans arrêt, sans arrêt [...] Je rêvais de mon entreprise avec des machines différentes. Je voyais mon matériel différent. Je voyais mes machines différentes. Ah oui, c'était toujours ça.

Elle s'exprimait également dans son activité mentale diurne marquant, sans pour autant s'y réduire, la volonté de Fernand d'installer l'objet aimé (son entreprise) au cœur de sa psyché.

Je ne pensais qu'à mon entreprise, jour et nuit, les dimanches et jours de fête. Cela n'a été presque que mes seuls sujets de conversation, sauf avec ma famille qu'ils ne comprenaient pas [F.C. parle ici de sa mère et de son frère]. Ils ne comprenaient pas qu'on ne puisse vivre que pour son travail.

Je pensais à faire mon travail, étudier le travail, les comptes de l'entreprise, son développement.

Je faisais de la facturation, je pensais au scénario de développement de l'entreprise. J'étais en machine, j'y pensais. Ça, c'était continuel. Il n'y avait jamais d'arrêt dans ce truc-là. Jamais. Je me réveillais même la nuit pour penser aux futures machines que $\mathrm{j}$ ' aurais pu amener dans mon entreprise et tout ce qui s'ensuit.

Cette forme de relation est proche de la forme d'identification que Tap (1979) appelle «l'identification de dépendance ou fusionnelle». L'ambiguïté de ce type de lien tient à l'ambivalence de la signification de l'objet pour le sujet : la relation est à la fois fusionnelle (superposition du sujet et de l'objet) et dissymétrique (dépendance plus ou moins totale du sujet à l'égard de l'objet qui satisfait ses besoins et désirs). 
Dans cette filiation symbolique aux ramifications multiples, l'entreprise était devenue pour Fernand un élément indispensable à son équilibre narcissique menacé et précaire, un élément vital dans la construction de son statut de sujet. Elle était pour lui un lieu privilégié de renégociation de son histoire (Dejours, 1987), un objet indispensable dans l'accomplissement de son désir (prouver sa valeur) qui lui permettait de relayer une souffrance venue du passé par des impératifs et des enjeux technico-économiques. Car si l'entreprise était pour Fernand un lieu de plaisir qu'il tirait de l'exercice de son activité professionnelle, elle était également un lieu de souffrance («La souffrance principale, c'était la fatigue. Je travaillais toujours fatigué. »). Cette souffrance était en partie liée à ce qu'il analyse aujourd'hui comme une surimplication professionnelle qui modifiait et fragilisait les frontières entre sa vie privée et sa vie professionnelle :

On vit cette passion avec des problèmes, des problèmes de couple toujours, et pourtant $\mathrm{j}$ 'avais une femme qui marchait dans le même sens que moi. Mais des problèmes de couple forcément parce qu'il n'y avait que le résultat de mon entreprise qui comptait et rien d'autre que mon entreprise.

De gros problèmes de couple parce que quand vous êtes quasiment parti une trentaine d'années de votre vie à partir de 5 heures du matin pour revenir vers les 10, 11 heures, minuit, 2 heures du matin tous les jours sauf le dimanche, ça finit par lasser votre partenaire.

Je n'étais jamais là. Mes gosses, je ne m'occupais jamais d'eux. Ma femme a supporté complètement la marche du ménage. Moi, ce que je demandais, c'est qu'on me foute la paix et d'aller travailler.

Ce surinvestissement (hérité de l'exemple paternel qui n'arrêta de travailler que quelques mois avant sa mort... à plus de 80 ans) était tel que Fernand ne cessera d'ailleurs son activité professionnelle que contraint et forcé par des problèmes cardiovasculaires alors qu'il se sentait à la limite de l'épuisement.

Je n'en pouvais plus de toute façon [...] J'ai arrêté parce que j'étais arrivé au fond de tout, j'étais usé de tout côté [...] J'ai changé par obligation parce que je n'étais plus capable de faire les choses [...] Je commençais à me voir vieillir. Je sentais que je n'avais plus autant de résistance au travail.

Ces difficultés de santé furent pour lui le déclic qui l'amena à cesser son activité professionnelle et à confier la direction de l'entreprise à son fils (qui possédait alors presque la totalité du capital). Cet arrêt a été rendu possible grâce au sentiment de confiance qu'il éprouve à l'égard de ce dernier pour garantir la pérennité de la firme.

J'ai une très grande confiance en Philippe [...] S'il n'avait pas assuré la pérennité, je ne l'aurais pas vécu comme ça. Je ne l'aurais pas vécu du tout comme ça. Je serais certainement retourné à l'atelier. 
Comme nous le montre la théorie de l'implication dans sa version psychoaffective (Thevenet, 1992), l'implication, qui peut s'apprécier à travers l'investissement de l'énergie dans la sphère professionnelle (Michel, 1989), peut s'analyser comme un processus d'identification de la personne à l'institution qui insiste sur les aspects affectifs de la relation individu-organisation. Le surinvestissement de Fernand dans son activité de travail était l'une des manifestations de son attachement affectif à son entreprise. Il faisait de sa participation à la vie de l'entreprise un facteur de définition personnelle. Indirectement, c'était également pour lui le moyen de conserver un lien de proximité quasi permanent avec son entreprise qu'il voyait comme l'unique moyen pour obtenir une confirmation affective positive de ses proches et surtout de sa mère.

\section{Le sentiment d'être dépositaire du destin de sa famille}

Tout individu est pris dans les mailles d'une filiation, porteur d'une histoire familiale qui inscrit ses effets de façon plus ou moins profonde dans ses membres en orientant, de façon consciente (par le processus de socialisation, Dubar, 1991) et inconsciente (par «l'encastrement de cryptes », Abraham et Torok, 1987), ses façons d'être, ses comportements et ses attitudes. Dans l'appropriation de cet héritage, les parents considèrent l'enfant comme le porteur de leurs rêves, de leurs désirs non réalisés à travers un contrat narcissique par lequel ce dernier est investi de cette mission d'avoir à assurer la continuité d'une mémoire générationnelle dont il se sent peu ou prou l'héritier. Comme le note Pagès (1993), il ne s' agit pas toujours d'une demande exprimée, et donc négociable ou opposable, mais d'une forme de contrat inconscient au niveau de l'être, et non du faire, qui lie l'enfant à l'une des figures parentales et par lequel celle-ci exerce une forme d'emprise sur les projets sociaux de l'enfant.

Ces influences transgénérationnelles apparaissent significatives dans l'histoire de vie de Fernand qui s'est toujours senti l'héritier d'une histoire familiale, senti investi d'une forme de responsabilité à l'égard du destin de sa famille.

Je gérais une famille complète avec son patrimoine. J'avais le truc de la famille sur le dos qu'il fallait impérativement sortir du trou. Il fallait sauver ma famille.

Renforcé dans sa détermination par la volonté de son père de le voir prendre sa succession, il s'est pleinement approprié un projet parental ${ }^{6}$ dont les finalités profondes correspondaient à un besoin de se conformer aux attentes de son père, à l'image, réelle ou imaginaire, qu'il renvoyait à cet homme idéalisé ( $«$ Je mettais mon père sur un piédestal, c'est-à-dire que c'était le père et le professeur. C'était tout pour moi. »).

6. Pour De Gaulejac (1991, p. 53), le projet parental désigne «l'ensemble des représentations que les parents se font de l'avenir de leurs enfants». 
C'est le papa qui m'a balancé cette idée-là. Parce que moi je ne voulais pas faire ce métier-là. Il me disait toujours : tu vas reprendre l'atelier...

Le sentiment de confiance que son père lui témoignait ne fera d'ailleurs que renforcer son intention de s'approprier et de réaliser le destin familial :

Ça nous a été dit que mon père avait confiance en moi et ma femme, Jacky, et ça nous avait conforté dans notre truc de dire «On va s'occuper de la famille ».

S'identifiant aux projections de son entourage, Fernand était prisonnier du désir de son père, inséré dans un ordre préétabli, attaché à un héritage tant matériel que symbolique le reliant à une généalogie qui faisait de lui le continuateur d'une histoire qu'il reproduisait à son tour. Dans une forme de contrat narcissique, son histoire se nourrissait d'identifications aliénantes l'empêchant de se dégager du pouvoir contraignant d'un projet parental vecteur de conflits (figure paternelle trop marquante ayant rendu la transmission difficile) et de se réapproprier son histoire personnelle. En ce sens, son destin était étroitement scellé à celui de son entreprise, à la nécessité d'avoir à assurer la continuité générationnelle. Cette firme constituait pour lui un prolongement de ses racines, la réalisation de son père qui l'avait créée en 1929, le fruit d'une vie entière de travail et de sacrifices (Levy, 1988).

Mon père a sauvé sa famille [...] Mon père, installant son entreprise en 1929, a sauvé sa famille des dettes [...] Il a resauvé cette famille en 1945 en rentrant de captivité en rachetant une maisonnette à mes grands-parents pour qu'ils puissent mourir en paix. Je reprends la famille à la suite de mon père, je resauve la famille et si c'était mon frère, lui, il la condamnait la famille. Il reperdait tout ce que mon père avait acquis, on balayait tous les sacrifices qu'il avait faits [...] car pour en arriver là où on en est, il a fallu le sacrifice de deux générations, mon père et la mienne.

Il a fallu à Fernand une détermination d'autant plus forte dans la réalisation de ce projet paternel qu'il s'opposait à une forte inertie de certains membres de sa famille, principalement celle de sa mère et de son frère. Sa mère était statutairement gérante de la société depuis la mort de son père en 1972 (qui était devenue alors une SARL), et ce, jusqu'en 1984 (même si Fernand assumait seul la direction de l'entreprise dans les faits). Durant cette même période, son frère cadet possédait $50 \%$ des parts sociales de l'entreprise et disposait d'une possibilité de blocage de toutes les décisions stratégiques, et notamment d'investissement.

J'avais un environnement familial qui mettait sur moi un couvercle, qui m'empêchait de me réaliser et qui empêchait la famille de se réaliser. C'était quelque chose d'épouvantable qui m'arrivait. Je combattais contre ça.

Je passais parfois des dimanches à pleurer de me sentir complètement coincé par une famille qui refusait tout ce que je proposais, j'en prenais entièrement la responsabilité, en aucun cas ils en auraient souffert. En aucun cas, ils en ont souffert. C'est terrible, vous savez! 
Il y avait un blocage de toute ma famille [...] un environnement qui m'a toujours été très défavorable [...] On a bagarré 28 ans. 28 ans de bagarre!

Malgré ces obstacles familiaux, la dimension symbolique de l'entreprise dans l'organisation psychique de Fernand et son attachement à celle-ci étaient tels qu'il n'a envisagé ni de quitter l'entreprise familiale pour créer une autre société, ni de saisir des occasions qui se sont présentées à deux reprises dans sa carrière (sans qu'il ne les sollicite lui-même) :

Je n'ai jamais pensé à cela. Je n'ai pensé qu'à une seule chose, c'est sauver l'entreprise C. [...] Ah oui, c'était celle-là ! J'étais capable de partir ailleurs. Mais non! Je voulais impérativement faire voir à ma famille que j'étais capable...

Je n'ai jamais cherché à quitter ma famille. C'était ma famille et mon entreprise d'abord!

\section{Discussion et conclusion}

La dynamique qui sous-tend la relation entre Fernand et son entreprise renvoie à des événements sociopsychiques s'inscrivant dans un temps «qui ne passe pas », c'est-à-dire qui se réfère «à des événements qui ne finissent pas, qui n'ont pas de terme et qui ne deviennent pas passés, mais qui ne demeurent pas immobiles et identiques pour autant » (Le Poulichet, 1994). Elle l'a conduit à vouloir combler ou réparer, de façon anachronique, des situations inachevées laissées en suspens dans un temps qui ne cesse pas (recherche d'un amour maternel) et à réaliser un projet paternel qui a donné un sens à sa vie (dans le double sens de signification et de direction). En d'autres termes, la nature du lien entre lui et son entreprise est fondée sur une relation d'attachement (prenant sens au regard de son parcours de vie) qui l'a conduit à rester prisonnier d'un certain nombre de déterminismes psychoaffectifs (recherche d'amour maternel) et sociofamiliaux (appropriation du projet paternel) dont la logique profonde lui échappait en grande partie (ce qu'il reconnaît d'ailleurs pleinement aujourd'hui). Dans un symbolisme paradoxal, la firme était simultanément une prothèse narcissique indispensable pour maintenir ses grands équilibres psychiques (étayages narcissiques, identité personnelle, image de soi, etc.) et un objet d'aliénation qui l'enfermait dans son histoire. Nous pouvons affirmer que Fernand vivait une relation d'attachement profondément investi sur le plan narcissique, affectif et symbolique avec son entreprise au regard de sa réaction lorsqu'il a dû faire face à la perte de cet « objet d'amour » ${ }^{7}$. En effet, il a vécu la transmission de son entreprise sur le mode d'une crise psychique pouvant être

7. D'une manière générale, l'attachement peut être défini comme «l'idée d'un lien affectif très fort, à des situations, états, signes, et finalement objets, lien par le moyen duquel le sujet accède au sentiment d'une existence propre» (Bianchi, 1989, p. 33). 
décrite par la théorie du deuil dont on retrouve clairement les étapes (Pailot, 1995, 1996). La théorie du deuil étant, par définition, une théorie de la perte d'un lien d'attachement qui remet en cause l'unité narcissique d'un sujet ${ }^{8}$, la reconnaissance d'un travail de deuil chez Fernand révèle et permet d'inférer la nature du lien de sens qui le liait à son entreprise.

Dans cette forme d'attachement viscéral, le dégagement rendu nécessaire par la succession managériale apparaît particulièrement complexe et difficile. Il se heurte à tout un ensemble de mécanismes de résistance et de défense qui s'opposent non seulement à une perte identitaire au niveau personnel et social ${ }^{9}$, mais aussi à une perte d'idéaux narcissiques qui soutenaient la structure de l'enveloppe psychique de Fernand depuis plusieurs décennies ${ }^{10}$. Dans ce cas, se dégager de ce lien d'attachement revient pour le dirigeant à s'extraire du mouvement qui avait donné sens à toute son existence. Transmettre l'entreprise lui demandait de faire le deuil impossible de désirs inachevés qu'il s'imaginait pouvoir réaliser par l'entremise de sa société. Ici, les enjeux de la succession managériale ne sont plus uniquement liés à la perte d'un pouvoir ou à une simple transformation identitaire liée à un changement de rôle social. Ils se réfèrent à ce que la personne a de plus profond en engageant son histoire et ses mémoires dans ce qu'elles ont de plus intimes.

La structuration du champ des études biographiques est marquée par la distinction entre l'ethnobiographie (individu considéré comme un miroir de son temps) et la psychobiographie (appréhension des composantes singulières qui éclairent l'histoire individuelle). Notre approche se situe bien évidemment dans le seconde perspective. Il est clair que cette forme de récit de vie est toujours idiosyncrasique tant dans son contenu que dans sa reconstruction. Cette remarque pose la question de la scientificité de la démarche. Au-delà des critiques adressées à l'étude de cas unique (difficulté de généralisation, confusion lorsque le cas est déviant, aspects descriptifs privilégiés sur les aspects théoriques, etc.), Gauthier (1992) et Yin (1989) considèrent que le recours au cas unique est justifié, notamment lorsqu'il permet d'observer et d'analyser un phénomène qui, jusqu' alors, était inaccessible à l'investigation scientifique (Yin, 1989). L'étude de cas que nous proposons ici nous paraît

8. Toute perte d'objet étant considérée, dans le cadre de cette théorie, comme une perte narcissique, c'est-à-dire une perte qui remet en cause le « ciment qui maintient l'unité constituée du Moi » (Green, 1983).

9. L'identité désigne, d'un point de vue objectif, l'ensemble des caractéristiques qui identifient un individu et, d'un point de vue subjectif, la conscience que chacun a de son individualité et la tendance à établir une continuité dans cette expérience subjective et à rechercher un sentiment d'unité et d'intégration, au-delà de la pluralité des rôles et des changements (Lipiansky, 1995).

10. Les idéaux narcissiques désignent « les représentations psychiques identificatoires [...] qui définissent pour l'individu une identité à partir de laquelle il peut progressivement s'affirmer comme un [moi, je] » (Duruz, 1985). 
répondre à ce critère. Certes, tous les dirigeants n'ont pas le même vécu, la même histoire que Fernand. Si nos résultats ne sont pas généralisables (mais quels sont les résultats qui le sont vraiment?), ils n'en permettent pas moins de donner une intelligibilité à un concept souvent utilisé par les gestionnaires sur un mode nominaliste mais rarement expliqué dans sa logique profonde, à savoir l'attachement ${ }^{11}$. Si l'on considère, comme Davis-Blake et Pfeffer (1989), que les chercheurs en sciences de l'organisation doivent réexaminer les problèmes conceptuels empiriques et pratiques de base qui affectent l'approche spécifique du comportement organisationnel $^{12}$, la méthode biographique ouvre des voies fécondes de théorisation. Elle peut se révéler pertinente chaque fois que l'on suppose que le passé d'un acteur organisationnel explique ses logiques d'action organisationnelles de manière plus pertinente que les éléments situationnels ou synchroniques. Au-delà de son inscription dans une logique interdisciplinaire (sans laquelle elle perd tout son intérêt), elle requiert toutefois un temps d'investigation et un niveau d'implication du narrateur que n'autorise pas toujours une recherche en gestion. Elle pose également la question délicate de l'interprétation du matériel biographique dans un cadre interdisciplinaire qui, sans confirmation externe, peut sombrer dans les méandres de la rhétorique et de l'herméneutique.

L'idée centrale de cette recherche était de montrer dans quelle mesure les freins psychologiques exprimés par certains dirigeants lors de la transmission de leur entreprise sont inséparables du lien de sens biographiquement construit qui les relie à cette dernière. Si l'analyse sociopolitique possède un pouvoir explicatif indéniable, elle n'offre pas un cadre théorique satisfaisant pour rendre compte de l'attachement viscéral de certains dirigeants pour leur entreprise, attachement qui reste pourtant un obstacle central (plus souvent évoqué qu'expliqué) lors des processus de transmission. Par son ouverture épistémologique, la méthode des récits de vie inscrite dans une perspective interdisciplinaire nous paraît être un cadre intéressant pour comprendre dans quelle mesure l'histoire de vie des dirigeants permet de donner une intelligibilité à certaines de leurs logiques d'action. Sans prôner une vision mécaniste de l'homme enfermé dans un déterminisme psychologique ou sociologique étroit, elle ne fait que prolonger la reconnaissance des zones d'intersection entre la famille et l'entreprise, particulièrement importantes dans l'analyse des entreprises familiales (Catry et Buff, 1996).

11. Rappelons, à ce titre, que l'attachement est un concept psychanalytique (d'ailleurs largement polysémique ; Pailot, 1995) dont il est bien malaisé de rendre compte par une analyse représentationnelle de surface.

12. Traduit de l'anglais must re-examine some of basic conceptual, empirical and practical problems that confront a dispositional approach to organizational behavior. 


\section{Bibliographie}

ABRAHAM, N. et M. TOROK (1987), L'écorce et le noyau, Paris, Flammarion (nouvelle édition révisée et complétée).

AMANN, B. et A. COURET (1992), «Les relations actionnaires-dirigeants selon les types d'organisation », Revue française de gestion, $\mathrm{n}^{\circ}$ 87, janvier-février, p. 93-102.

ARON, R. (1989), Leçons sur l'histoire, Paris, Éditions de Fallois, p. 157.

BAUER, M. (1993), Les patrons de PME entre le pouvoir, l'entreprise et la famille, Paris, InterÉditions, Collection «L'Entreprise ».

BAUMERT, H. (1992), Succession dans la PME familiale, Paris, Les Éditions d'Organisation, Collection «PME».

BERnOUX, P. (1995), La sociologie des entreprises, Paris, Éditions du Seuil, p. 69.

BerTAuX, D. (1997), Les récits de vie, Paris, Éditions Nathan.

BIANCHI, H. (1989), Vieillir ou les destins de l'attachement, Paris, Dunod, Collection «La question du vieillissement ».

Blanchet, A. (1983), «Épistémologie critique de l'entretien d'enquête de style non directif », Bulletin de Psychologie, $\mathrm{n}^{\circ} 358$, tome 36, p. 352-372.

Boudon, R. (1986), L'idéologie ou l'origine des idées reçues, Paris, Éditions du Seuil.

BOURDIEU, P. (1986), «L'illusion biographique », Actes de recherches en sciences sociales, $\mathrm{n}^{\text {os }}$ 62-63, juin, p. 69-72.

Bourdieu, P., J.-C. ChAmberon et J.-C. PASSERON (1973), Le métier de sociologue; préalables épistémologiques, Paris, Mouton Éditeur (deuxième édition révisée), p. 14.

CARLAND, J.W., F. HOY, W.R. Boulton et J.C. CARLAN (1984), « Differentiating entrepreneurs from small business owners : a conceptualization », Academy of Management Review, vol. 9, $\mathrm{n}^{\circ}$ 2, p. 354-359.

CATRY, B. et A. Buff (1996), Le gouvernement de l'entreprise familiale, Paris, PubliUnion Éditions.

Clapier-Valladon, S. (1983), «Le récit de vie», Bulletin de Psychologie, $\mathrm{n}^{\circ}$ 361, tome 36.

Clapier-VAlladon, S. et P. RAyBAut (1993), Les récits de vie : théorie et pratique, Paris, Presses universitaires de France, Collection « Le sociologue » ( $3^{e}$ édition mise à jour).

CORCUFF, P. (1995), Les nouvelles sociologies, Paris, Nathan Université.

CROUZET, F. (1995), « Rôle et géographie des dynasties d'affaires », Entreprises et Histoire, no 9, p. 5-11.

Crozier, M. et E. Friedberg (1977), L'acteur et le système, Paris, Éditions du Seuil.

DAVIS-BLAKE, A. et J. PFEFFER (1989), « Just a mirage : the search for dispositional effects in organizational research ", Academy of Management Review, vol. 14, $\mathrm{n}^{\circ} 3$, p. 385-400.

De GaUleJAC, V. (1984), « Approche socio-psychologique des histoires de vie », Éducation Permanente, $\mathrm{n}^{\text {os }}$ 72-73, p. 30-47. 
De Gaulejac, V. (1991), La névrose de classe, Paris, Hommes et Groupes Éditeurs ( 2 édition corrigée).

DEJOuRs, C. (sous la direction de) (1987), Plaisir et souffrance dans le travail, Paris, publié avec le concours du CNRS, tomes 1 et 2 .

DEJOURS, C. (1993), Travail, usure mentale : de la psychopathologie à la psychodynamique du travail, Paris, Bayard Éditions (nouvelle édition augmentée).

DUBAR, C. (1991), La socialisation : construction des identités sociales et professionnelles, Paris, Armand Colin.

DuCHÉNAUT, B. (1995), Enquête sur les PME françaises, Paris, Maxima, Laurent du Mesnil Éditeur.

DuCHÉNAUT, B. (1996), Les dirigeants de PME, Paris, Maxima, Laurent du Mesnil Éditeur.

DuruZ, N. (1985), Narcisse en quête de soi : étude des concepts de narcissisme, de moi et de soi en psychanalyse et en psychologie, Bruxelles, Pierre Mardaga Éditeur, Collection «Psychologie et sciences humaines», p. 137.

ENRIQUEZ, E. (1991), Les figures du Maître, Paris, Arcantère Éditions.

FERRAROTTI, F. (1983), Histoire et histoires de vie: La méthode biographique dans les sciences sociales, Paris, Méridiens Klincksieck.

GAUltier, A. (1980), Pérennité et succession dans les entreprises moyennes familiales, Paris, Éditions Hommes et Techniques.

GAUTHIER, B. (1992), «La structure de preuve», dans B. Gauthier (sous la direction de), Recherche sociale : de la problématique à la collecte des données, Sainte-Foy, Presses de l'Université du Québec.

GÉLINIER, O. et A. GAULTIER (1974), L'avenir des entreprises personnelles et familiales, Paris, Éditions Hommes et Techniques.

GOMEZ, P.-Y. (1996), Le gouvernement de l'entreprise : modèles économiques de l'entreprise et pratiques de gestion, Paris, InterÉditions.

GREEN, A. (1983), Narcissisme de vie, narcissisme de mort, Paris, Éditions de Minuit, Collection «Critique».

HiRIGOYEN, B. (1987), « La transmission des PME familiales : constat et suggestions », La Revue du Financier, $n^{\circ}$ 55, p. 37-48.

KETS DE VRIES, M. (1988), «Succession du PDG : l'ombre au tableau », Harvard L'Expansion, $\mathrm{n}^{\mathrm{o}} 50$, automne, p. 99-104.

LANGLOIS, C. (1987), Succession du chef d'entreprise : contribution à l'étude de transmission de la petite entreprise familiale, Thèse de doctorat de $3^{\mathrm{e}}$ cycle, IAE et USTL de Lille, avril.

LEGRAND, M. (1993), L'approche biographique : théorie, clinique, Paris, Hommes et Perspectives.

LEHMANN, P.-J. (1993), «Le financement de la transmission des PME», Revue française de gestion, $\mathrm{n}^{\circ}$ 95, septembre-octobre, p. 80-102.

Le Poulichet, Sylvie (1994), L'ouvre du temps en psychanalyse, Paris, Éditions Payot et Rivages, Collection « Rivages / Psychanalyse », p. 48. 
LEVY, A. (sous la direction de) (1988), Les dirigeants de PME et leur entreprise, Recherche effectuée pour le ministère de la Recherche, GRAD, Université d'Angers, mai.

LIPIANSKY, E.M. (1995), « L'identité à l'articulation du psychologique et du social », Revue internationale de psychosociologie, vol. 2, $\mathrm{n}^{\circ}$ 2, p. 21-34.

Michel, S. (1989), Peut-on gérer les motivations?, Paris, Presses universitaires de France, Collection «Gestion».

MuCCHIELLI, A. (1991), Les méthodes qualitatives, Paris, Presses universitaires de France, Collection «Que sais-je ?», n 2591.

MuCCHIELLI, A. (1993), L'analyse formelle des rêves et des récits d'imagination, Paris, Presses universitaires de France, Collection «Le psychologue».

PAGÈs, M. (1993), Psychothérapie et complexité, Paris, Hommes et Perspectives.

PAILOT, P. (1995), L'articulation entre l'évolution de la firme et l'évolution du dirigeant dans un contexte de changement: de l'emprise au deuil-approche qualitative inductive basée sur la méthode biographique, Thèse de doctorat, Université des Sciences et Technologies de Lille I, novembre.

PAILOT, P. (1996), « Réflexions sur les conditions d'application de la théorie du deuil en sciences de gestion », Actes des $6^{e s}$ Journées nationales d'études psychanalyse et management, Institut Psychanalyse et Management, Groupe ESC Nantes, 23-24 mai, p. 30-52.

PAQUEROT, M. (1996), «L'enracinement des dirigeants et ses effets », Revue française de gestion, $\mathrm{n}^{\circ} 111$, novembre-décembre, p. 212-225.

PASSERON, J.-C. (1991), Le raisonnement sociologique : l'espace non-poppérien du raisonnement naturel, Paris, Nathan.

PENEFF, J. (1990), La méthode biographique : de l'École de Chicago à l'histoire orale, Paris, Armand Colin Éditeur.

Pineau, G. et J.-L. Le GRAND (1993), Les histoires de vie, Paris, Presses universitaires de France, Collection «Que sais-je ? », $\mathrm{n}^{\circ} 2760$.

SMITH, L.M. (1994), « Biographical method», dans N.K. Denzin, Y.S. Lincoln (dir.), Handbook of Qualitative Research, chapitre 18, Newbury Park, California, Sage Publication Inc., p. 286-305.

TAP, P. (1979), «L'identification est-elle une aliénation de l'identité ? », dans P. Tap (sous la direction de), Identité individuelle et personnalisation, Toulouse, Privat, Collection «Sciences de l'Homme».

THEVENET, M. (1992), Impliquer les personnes dans l'entreprise, Paris, Éditions Liaisons.

VATTEVILLE, E. (1994), «Le risque successoral », Revue française de gestion, $\mathrm{n}^{\circ}$ 98, marsavril-mai, p. 18-27.

VOYDEVILLE, D. (1992), « Transmissions d'entreprises à titre gratuit », La Revue Banque, $n^{\circ} 531$, octobre, p. 45-61.

WACHEUX, F. (1996), Méthodes qualitatives et recherche en gestion, Paris, Economica, Collection «Gestion », Série Politique générale, finance, marketing.

WIDLÖCHER, D. (1981), « Genèse et changement », Revue française de psychanalyse, vol. 4. 
YIN, R. (1981), « The case study crisis : some answers », Administrative Science Quarterly, $\mathrm{n}^{\mathrm{0}} 26$, p. 58-65.

YIN, R. (1989), Case Study Research: Design and Methods, Applied Social Research Methods Series, vol. 5, Newbury Park, California, Sage Publications Inc., édition révisée.

ZALEZNIK, A. (1994), Les ressorts de l'action, Paris, InterÉditions, p. 144. 